\title{
Post-hysterectomy intravenous leiomyomatosis: A case of successful multidisciplinary surgery under non-extracorporeal circulation
}

\author{
YUCUI ZENG ${ }^{1}$, HUIRU TANG $^{1}$, LIPING ZENG $^{1}$, LIHUI WEI ${ }^{2}$, XIAOMING ZHANG $^{2}$ and RUIFANG WU ${ }^{1,3}$ \\ ${ }^{1}$ Department of Gynecology and Obstetrics, Peking University Shenzhen Hospital, Shenzhen, \\ Guangdong 518036; ${ }^{2}$ Department of Gynecology and Obstetrics, Peking University Second Hospital, \\ Beijing 100044; ${ }^{3}$ Shenzhen Technical Research and Development Center on Gynecologic Oncology, \\ Peking University Shenzhen Hospital, Shenzhen, Guangdong 518036, P.R. China
}

Received May 12, 2016; Accepted August 30, 2016

DOI: $10.3892 /$ mco.2016.1074

\begin{abstract}
Intravenous leiomyomatosis is a rare benign disease. We here in present the case of a 39-year-old woman with a history of hysterectomy who presented with intermittent abdominal pain, palpitations and tightness of the chest. Physical examination revealed the presence of a pelvic mass of regular shape. Gynecological ultrasonography, computed tomography scans and three-dimensional (3D) cardiac ultrasonography were used to evaluate the imaging characteristics of the mass and reach a final diagnosis. The mass appeared to extend to the iliac veins, renal veins and inferior vena cava on imaging examination. The mass was successfully excised under non-extracorporeal circulation in one stage. Pathological examination of tumor samples indicated intravenous leiomyomatosis. After the operation, the symptoms were dissipated and no abnormal echo was observed in the inferior vena cava or the right atrium on 3D-cardiac ultrasonography. The patient is currently followed up without signs of recurrence. The aim of the present study was to describe in detail the diagnostic procedure and treatment in order to improve our current understanding of this disease.
\end{abstract}

\section{Introduction}

Intravenous leiomyomatosis is a rare disease originating from myometrial veins, characterized by intravascular nodular masses histologically composed of benign smooth muscle cells; the masses may extend to a variable distance into the inferior vena cava, right atrium and even the right ventricle.

Correspondence to: Dr Ruifang Wu, Department of Gynecology and Obstetrics, Peking University Shenzhen Hospital, 1120 Lianhua Road, Futian, Shenzhen, Guangdong 518036, P.R. China

E-mail:wurfpush@126.com

Key words: intravenous leiomyomatosis, hysterectomy, non-extracorporeal circulation
It is reported that $>50 \%$ of the patients have a prior history of hysterectomy (1). These nodules may extend into the atrium and/or ventricle and may be accompanied by clinical symptoms such as breathlessness, pain and congestive myocardial infarction, and they may be misdiagnosed as cardiac myxoma. To date, the treatments for intravenous leiomyomatosis include expectant treatment and surgical resection. Gonadotropin-releasing hormone analogue (GnRHa) is important in expectant treatment. However, the outcome of such cases is generally not satisfactory. Surgical management includes complete and incomplete resection. We here in present a case of diffuse uterine leiomyomatosis extending to the right atrium, which was successfully resected under non-extracorporeal circulation.

\section{Case report}

A 39-year-old woman, gravida 2, para 2, presented with complaints of intermittent abdominal pain over the last 3 years, with palpitations and tightness of the chest over the last 2 months.

In March, 2012, the patient underwent uterine myomectomy in the Jiangxi Provincial People's Hospital. In January, 2013, the patient was admitted to the Chenzhou City People's Hospital and underwent panhysterectomy with left adnexectomy. Detailed information on the abovementioned surgeries were not available. One year later, the patient presented to the Chenzhou First People's Hospital due to a recurrent abdominal mass with abdominal distention, was diagnosed with disseminated intravenous leiomyomatosis, and three cycles of GnRHa were administered. In May, 2014, the patient presented to the Peking University Shenzhen Hospital (Shenzen, China) for treatment, and was prescribed triptorelin acetate injections (3.75 mg) every 28 days. However, the patient did not follow the doctor's instructions and the injections were performed in May, June, August and October. In December, 2014, the symptoms were aggravated and were accompanied by edema of the bilateral lower extremities. On general physical examination, the mass occupied the entire pelvis, resembling a 4-month pregnancy. The patient was again administered 
Table I. Results of gynecological ultrasonography examination of the patient in our hospital.

\begin{tabular}{lcl}
\hline Date (year/month) & Pelvic mass size $(\mathrm{mm})$ & Mass description \\
\hline $2014 / 03$ & $72 \times 45 \times 68$ & Dispersive distribution, different sizes \\
$2014 / 07$ & $68 \times 53$ & Mixed mass \\
$2014 / 10$ & $81 \times 76 \times 64$ & Mixed mass \\
$2014 / 12$ & $120 \times 110$ & Multiple hyperechoic masses, blood flow signals \\
$2015 / 04$ & $104 \times 67 \times 100$ & Multiple mass integration, irregular shape, streak \\
& & blood flow signals
\end{tabular}
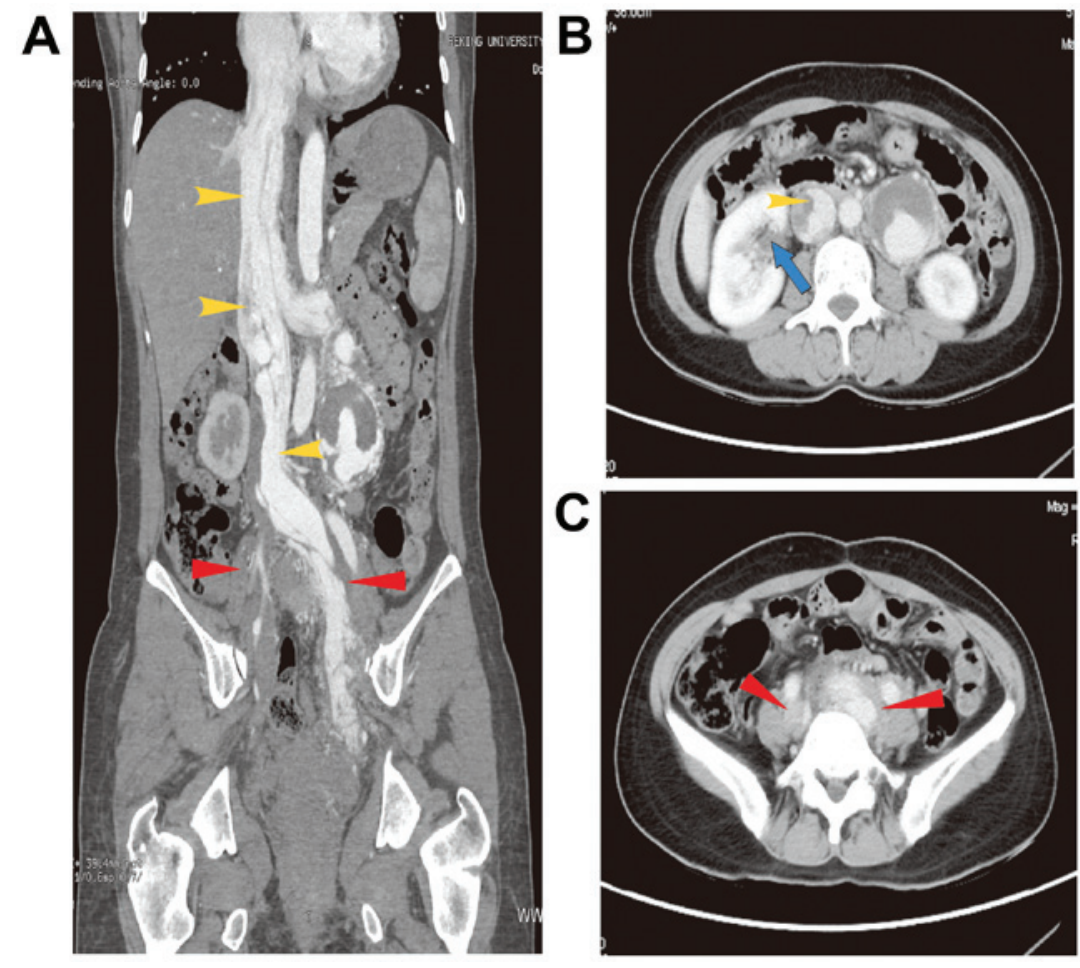

Figure 1. Computer tomography scan images showing (A) the extent of the tumor mass (yellow arrowheads, inferior vena cava; red arrowheads, bilateral iliac veins); (B) the mass in the inferior vena cava (yellow arrowhead) and renal veins (blue arrow); and (C) the mass in the bilateral iliac veins (arrowheads).

3.75-mgtriptorelin acetate injections in December, 2014 and January, March and April, 2015. The patient has received a total of 11 cycles of GnRHa to date.

The patient presented to our hospital with a history of chest tightness and palpitations for 2 months. On physical examination, a grade III systolic murmur was audible along the left edge of the sternum. Pelvic examination revealed bilateral pelvic solid masses sized 10x10 (left side) and 3x4 (right side) $\mathrm{cm}$, hard on palpation, irregular, immobile and non-tender; the left mass had reached the left pelvic wall and was fixed to the obturator foramen. As the patient had first presented to our hospital, gynecological ultrasonography examination revealed the changes in the pelvic mass (Table I). Computed tomography scans revealed pelvic mass extension to the bilateral internal and common iliac veins, left renal vein and inferior vena cava, with a small-to-low-density lesion in the liver (Fig. 1). Three-dimensional (3D) cardiac ultrasonography revealed that the wide inner diameter of the inferior vena cava was $28 \mathrm{~mm}$, with a diffuse mixed echo; a low echo was mainly visible in the inferior vena cava (unclear boundary, irregular shape, with strong streak-like echogenicity), partly extending to the right atrium $(28 \times 24 \mathrm{~mm})$, with a small amount of tricuspid valve regurgitation (Fig. 2A-C).

Based on the patient's history and the abovementioned examinations, intravenous leiomyomatosis was diagnosed. Following a multidisciplinary discussion, the patient underwent myomectomy (right atrium, inferior vena cava, renal veins and pelvis), right oophorectomy and pelvic adhesiolysis under non-extracorporeal circulation. The length of the tumor from the inferior vena cava to the right atrium was $30 \mathrm{~cm}$; the tumors in the bilateral renal veins were 6 and $5 \mathrm{~cm}$, respectively; the tumor in the right common iliac vein was sized $12 \times 4 \mathrm{~cm}$; the tumor in the left common iliac vein to the left pelvis was $8 \times 7$ and $10 \mathrm{~cm}$ in length; and the right ovary was sized $3 \times 2.5 \times 1.5 \mathrm{~cm}$ (Fig. 3). The postoperative pathological examination indicated intravenous leiomyomatosis (Fig. 4). Follow-up 3D-cardiac ultrasonography revealed no visible echo in the inferior vena cava or the right atrium (Fig. 2D). 

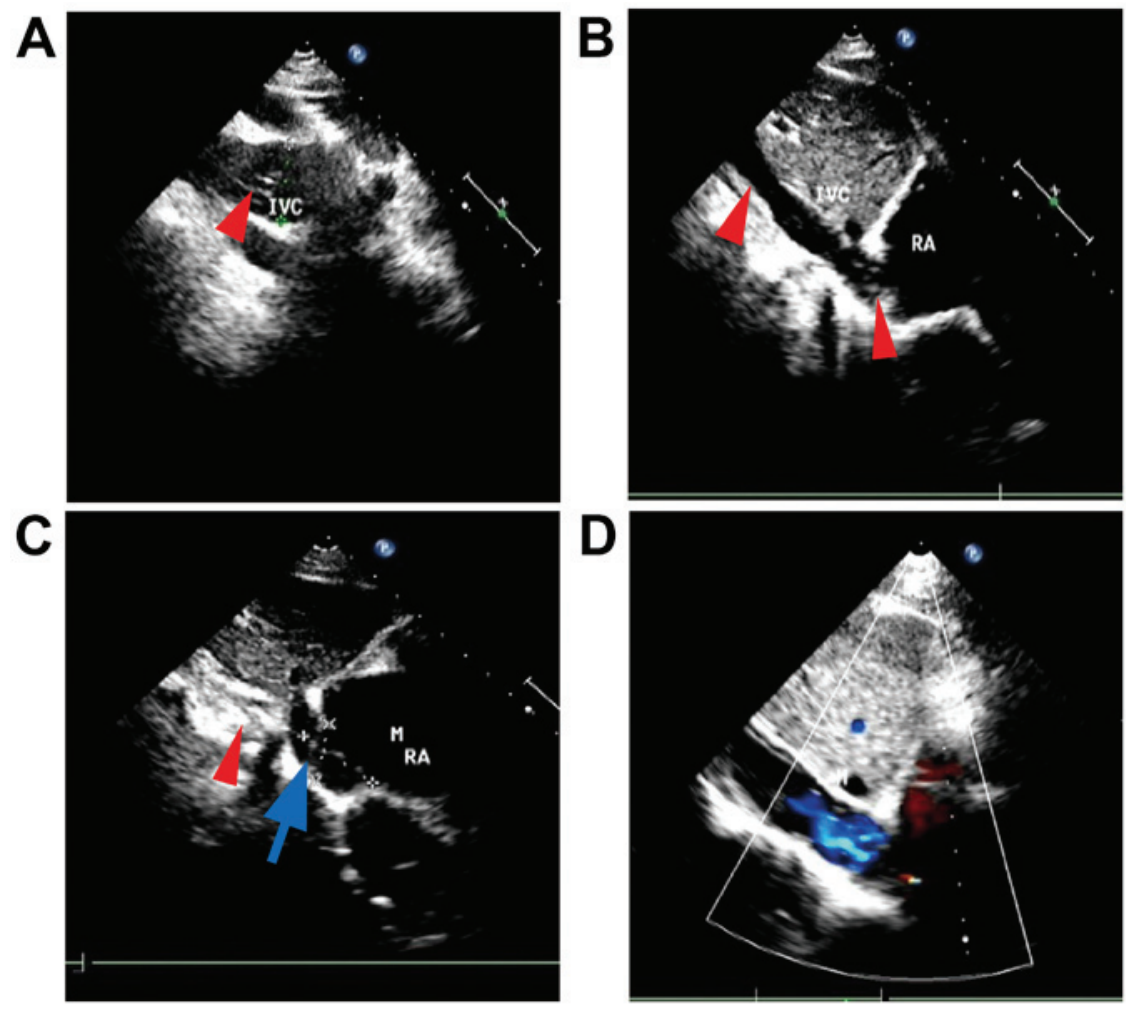

Figure 2. Three-dimensional cardiac ultrasonography before and after the operation. (A) Strong streak-like echo in the inferior cava vena (IVC; arrowhead). (B) Tumor extension to the right atrium (RA; arrowheads). (C) The tumor size in the RA was $28 \times 24 \mathrm{~mm}$ (blue arrow); the red arrowhead shows a strong echo in the IVC. (D) There was no abnormal echo in the IVC and RA after the operation.
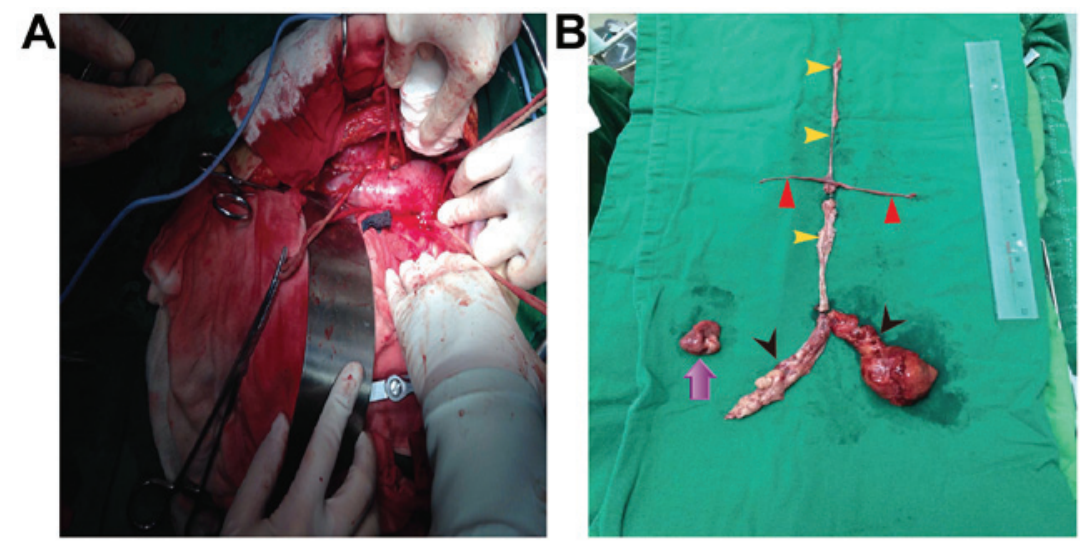

Figure 3. (A) Operative field. (B) Excised tumor sample consisting of a mass in the inferior vena cava and right atrium (yellow arrowheads), a mass in the bilateral renal veins (red arrowheads), a mass in the bilateral iliac veins (black arrowheads) and the right ovary (purple arrow).
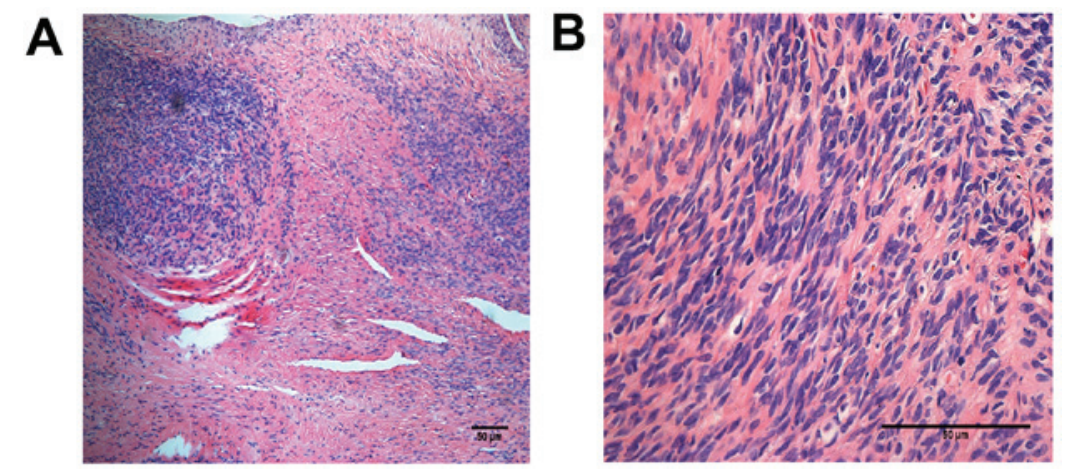

Figure 4. Pathological examinationof the intravenous mass; hematoxylin and eosin staining, magnification (A) x100 and (B) x400. (A) The tumor cells were densely packed. (B) The mass consisted of spindle cells arranged in whorls, without cytological atypia, mitotic figures, or necrosis. 


\section{Discussion}

The etiology of intravenous leiomyomatosis remains unclear, but two theories have been proposed: One suggests that intravenous leiomyomatosis originates from smooth muscle cells in the vessel wall, whereas the other suggests that intravenous leiomyomatosis arises from a uterine leiomyoma, with the benign tumor cells invading the uterine veins and continuing to grow along the venous circulation $(2,3)$.

Ordulu et al (4) attempted to explain the pathogenesis of intravenous leiomyomatosis by molecular cytogenetic analyses, and they suggested that dysregulation of the non-histone chromatin-associated architectural factor HMGA2, which affects the differentiation and proliferation at $12 \mathrm{q} 14$, plays a role in the development of intravenous leiomyomatosis. Leiomyomatosis peritonealis disseminata (LPD) is a subtype of intravenous leiomyomatosis that usually occurs in women of reproductive age. Yuri et al (5) reported that LPD lesions expressed progesterone receptor, while they were negative for estrogen receptor and luteinizing hormone receptor expression. Kokawa et al (6) indicated that high levels of estradiol were associated with the development of intravenous leiomyomatosis.

Intravenous leiomyomatosis extending to the atrium may be confused with intracardiac tumors, such as myxoma and lipoma, or thrombus formation, and cause multiple symptoms, such as chest pain, breathlessness and syncope. Computed tomography (CT) images may help identify lesions in the inferior vena cava. However, as a proportion of the patients are reportedly asymptomatic, it is crucial to make an early accurate diagnosis and select the appropriate treatment schedule.

The majority of the patients have a history of uterine leiomyoma or hysterectomy. Imaging is also important for correct diagnosis. Gui et al (7) reported that CT angiography may reveal the location, size and full-scale extension pathway of intravenous leiomyomatous lesions, and maybe used as the first-line imaging modality in preoperative assessment. When leiomyomatosis affects the spine, magnetic resonance imaging may provide information for the diagnosis and the extent of the lesions (8). Echocardiography with good penetration of the tumor is also helpful in reaching a diagnosis (9).

There are currently no established guidelines regarding the treatment of intravenous leiomyomatosis. However, therapy must be individualized according to the patients' age, hormonal and reproductive status and symptomatology. Surgery is the only effective treatment for intravenous leiomyomatosis extending to the inferior vena cava and the cardiac chambers. Surgical treatment includes one-stage or two-stage surgery.

In the present case, we applied a series of successful one-stage surgeries; the pelvic and chest surgeries were performed at the same time. The type of surgery performed should be also based on the patients' general condition and the size of the tumor. Most researchers use cardiopulmonary bypass when excising the mass in the inferior vena cava or the right atrium; however, this is associated with an increased risk of ischemia and perfusion injury, oxidative stress injury of vital organs (e.g., acute lung injury and kidney injury) and thrombogenesis $(10,11)$. In the present case, we performed the surgery under non-extracorporeal circulation, which may decrease non-physiological alterations and postoperative complications, but may also increase the degree of difficulty of the operation. Venous return was controlled by the bilateral pinch-off method over a short time period (mean, 3-4 min). This treatment may provide a reference for other clinicians, as successfully performing this surgery under non-extracorporeal circulation was proven to be feasible.

A total of 11 cycles of GnRHa was administered prior to the operation in this case, although the efficacy of hormonal therapy (GnRHa) was questionable. However, a previous study reported that GnRHa therapy following surgery in LPD may prevent the recurrence of new lesions (12). Evidence of long-term efficacy of postoperative treatment in intravenous leiomyomatosis is lacking, and further investigation is required. Doyle et al (13) reported that aromatase inhibitors are effective in preventing tumor progression and recurrence in patients with incompletely resected intravenous leiomyomatosis with cardiac extension.

Postoperative patient follow-up is required, as recurrence is frequent. Hereditary leiomyomatosis and renal carcinoma (HLRCC) syndrome is an autosomal dominant syndrome that results from mutations in the fumarate hydratase gene (14). The fumarate hydratase gene is located on a highly conserved region of the 1q42.3-43 chromosome (15). Patients with HLRCC are commonly aged 10-44 years (although genetic testing should be offered to children as young as 8-10 years of age) and at risk of uterine smooth muscle tumors, as well as renal tumors (16). Therefore, it is imperative to investigate patient history, and follow-up should include evaluation of recurrence of the primary disease and occurrence of correlative renal tumors.

One-stage resection was successfully completed in this case under non-extracorporeal circulation. The diagnosis of intravenous leiomyomatosis requires imaging combined with clinical manifestations and history. Surgery is the standard treatment once the diagnosis is established. Tissue biopsy is a definitive method for diagnosing intravenous leiomyomatosis. The follow-up should be started immediately after the hysterectomy in order to timely detect early-stage intravenous leiomyomatosis.

\section{Acknowledgements}

The authors appreciate the assistance of Dr Zhang Xiaoming and Dr Wei Lihui (Peking University People Hospital) with directing the surgery. The present study was supported by grants from the Shenzhen Municipal Science and Technical Innovation Committee, Shenzhen Technical Research and Development Center on Gynecologic Oncology (no. GCZX2015043016200372), the Science and Technology Planning Project of Guangdong Province (no. 2013B021800095), and the Science and Technology Planning Project of Shenzhen Municipal Government (no. JCYJ20140415162338852).

\section{References}

1. Mizuno T, Mihara A and Arai H: Intracardiac and intravascular leiomyomatosis associated with a pelvic arterio-venous fistula. Ann Transl Med 2: 48, 2014.

2. Kutay V, Tuncer M, Harman M, Ekim H and Yakut C: Intracardiac extension of intravenous leiomyoma. Texas heart institute journal/from the Texas heart institute of St. Luke's episcopal hospital, Texas children's hospital 32: 232-234, 2005. 
3. Nam MS, Jeon MJ, Kim YT, Kim JW, Park KH and Hong YS Pelvic leiomyomatosis with intracaval and intracardiac extension: A case report and review of the literature. Gynecol Oncol 89: $175-180,2003$

4. Ordulu Z, Nucci MR, Dal Cin P, Hollowell ML, Otis CN, Hornick JL, Park PJ, Kim TM, Quade BJ and Morton CC: Intravenous leiomyomatosis: An unusual intermediate between benign and malignant uterine smooth muscle tumors. Mod Pathol 29: 500-510, 2016

5. Yuri T, Kinoshita Y, Yuki M, Yoshizawa K, Emoto Y and Tsubura A: Leiomyomatosis peritonealis disseminata positive for progesterone receptor. Am J Case Rep 16: 300-304, 2015.

6. Kokawa K, Yamoto M, Yata C, Mabuchi Y and Umesaki N: Postmenopausal intravenous leiomyomatosis with high levels of estradiol and estrogen receptor. Obstet Gynecol 100: 1124-1126, 2002.

7. Gui T, Qian Q, Cao D, Yang J, Peng P and Shen K: Computerized tomography angiography in preoperative assessment of intravenous leiomyomatosis extending to inferior vena cava and heart. BMC Cancer 16: 73, 2015.

8. Hur JW, Lee S, Lee JB, Cho TH and Park JY: What are MRI findings of Spine Benign Metastasizing Leiomyoma? Case report with literature review. Eur Spine J 24 (Suppl 4): S600-S605, 2015.

9. Li R, Shen Y, Sun Y, Zhang C, Yang Y, Yang J, Su R and Jiang B: Intravenous leiomyomatosis with intracardiac extension: Echocardiographic study and literature review. Tex Heart Inst J 41: 502-506, 2014
10. Yang FY, Bao YZ, Liu FS, Zhu YC, Zheng J,Zhang JH,Zheng XF and Wei GC: Non-extracorporeal circulation for coronary artery bypass graft surgery is more beneficial than extracorporeal circulation. Eur Rev Med Pharmacol Sci 19: 1452-1456, 2015.

11. Zhang YH, Jin CZ, Jang JH and Wang Y: Molecular mechanisms of neuronal nitric oxide synthase in cardiac function and pathophysiology. J Physiol 592: 3189-3200, 2014.

12. Bisceglia M,Galliani CA,Pizzolitto S, Ben-Dor D, Giannatempo G, Bergoli AL and Aieta M: Selected case from the Arkadi M. Rywlin international pathology slide series: Leiomyomatosis peritonealis disseminata: Report of 3 cases with extensive review of the literature. Adv Anat Pathol 21: 201-215, 2014.

13. Doyle MP, Li A, Villanueva CI, Peeceeyen SC, Cooper MG, Hanel KC, Fermanis GG and Robertson G: Treatment of intravenous leiomyomatosis with cardiac extension following incomplete resection. Int J Vasc Med 2015: 756141, 2015.

14. Joseph NM, Solomon DA, Frizzell N, Rabban JT, Zaloudek C and Garg K: Morphology and immunohistochemistry for 2SC and $\mathrm{FH}$ aid in detection of fumarate hydratase gene aberrations in uterine leiomyomas from young patients. Am J Surg Pathol 39: 1529-1539, 2015.

15. Bayley JP, Launonen V and Tomlinson IP: The FH mutation database: An online database of fumarate hydratase mutations involved in the MCUL (HLRCC) tumor syndrome and congenital fumarase deficiency. BMC Med Genet 9: 20, 2008.

16. Mann ML,Ezzati M, Tarnawa ED and Carr BR: Fumarate hydratase mutation in a young woman with uterine leiomyomas and a family history of renal cell cancer. Obstet Gynecol 126: 90-92, 2015. 